ISSN-L 2077-0014

DOI: https://doi.org/10.33326/26176068.2021.3.1152

Artículo original/Original article/Artigo original

\title{
Calidad de sueño en estudiantes de la Facultad Ciencias de la Salud en una universidad pública peruana
}

\author{
Sleep quality in students of the Faculty of Health Sciences in a Peruvian public university \\ Qualidade de sono em estudantes da Faculdade Ciências da Saúde de uma universidade pública peruana \\ María del Carmen Silva-Cornejo ${ }^{1}$ \\ (D) https://orcid.org/0000-0002-2695-0659
}

\begin{abstract}
Resumen
Objetivo: Identificar la calidad de sueño de los estudiantes de la Facultad de Ciencias de la Salud. Material y métodos: Estudio descriptivo de corte transversal, realizado en la Facultad de Ciencias de la Salud de la Universidad Nacional Jorge Basadre Grohmann de Tacna, en 153 estudiantes de las 5 escuelas profesionales, seleccionados de forma aleatoria simple. Se aplicó cuestionario de calidad de sueño de Pittsburgh. Resultados: La calidad subjetiva de sueño de los estudiantes fue buena $(47,1 \%)$ y mala $(42,5 \%)$, la latencia de sueño presenta dificultad leve $(45,1$ $\%)$ y moderada (32\%), la duración del dormir fue mayor de 5 a 6 horas $(43,1 \%)$, le sigue menos de 5 horas $(26,1$ $\%)$, la eficiencia habitual del sueño es menos del $65 \%$ con un porcentaje de $90,8 \%$, las alteraciones del sueño se clasifica en normal $(50,3 \%)$ y diurna marginal $(46,4 \%)$, la disfunción diurna es moderadamente $(45,1 \%)$ y ligeramente problemático $(35,9 \%)$, el nivel de perturbación de sueño merece atención médica y tratamiento en $81,7 \%$, la mayoría de los estudiantes merecen atención y tratamiento médico: Enfermería $19 \%$ y Odontología 17,6 \%. Conclusión: Las carreras profesionales que tienen nivel de perturbación de sueño y merecen atención y tratamiento médico en orden decreciente son Enfermería (19\%), Odontología (17,6 \%), Farmacia y Bioquímica (16,3\%), Obstetricia (15\%) y Medicina $(13,7 \%)$.
\end{abstract}

Palabras clave: calidad de sueño, estudiantes de ciencias de la salud, sueño

\begin{abstract}
Objective: To identify the quality of sleep of the students of the Faculty of Health Sciences. Material and methods: Descriptive cross-sectional study, carried out at the Faculty of Health Sciences of the Jorge Basadre Grohmann National University of Tacna, in 153 students from the 5 professional schools, selected in a simple random way, Pittsburgh sleep quality questionnaire was applied. Results: The subjective sleep quality of the students was good (47.1\%) and bad (42.5\%), sleep latency presents mild (45.1\%) and moderate (32\%) difficulty, sleep duration was greater than 5 to 6 hours $(43.1 \%)$, followed by less than 5 hours $(26.1 \%)$, usual sleep efficiency is less than $65 \%$ with a percentage of $90.8 \%$, sleep disturbances are classified as normal (50.3\%) and marginal daytime (46.4\%), daytime dysfunction is moderate $(45.1 \%)$ and slightly problematic $(35.9 \%)$, the level of sleep disturbance deserves medical attention and treatment in $81.7 \%$, the majority of the students deserve medical attention and treatment: Nursing $19 \%$ and Dentistry $17.6 \%$. Conclusion: The professional careers that have a level of sleep disturbance and deserve medical attention and treatment in decreasing order are Nursing (19\%), Dentistry (17.6\%), Pharmacy and Biochemistry (16.3\%), Obstetrics $(15 \%)$ and Medicine (13.7 \%).
\end{abstract}

Keywords: health science students, sleep, sleep quality

${ }^{1}$ Universidad Nacional Jorge Basadre Grohmann. Doctora en Ciencias. Docente de la Escuela Profesional de Enfermería. Tacna, Perú 


\section{Resumo}

Objetivo: Identificar a qualidade de sono dos estudantes da Faculdade de Ciências da Saúde. Material e métodos: Estudo descritivo de corte transversal, realizado na Faculdade de Ciências da Saúde da Universidade Nacional Jorge Basadre Grohmann de Tacna, em 153 estudantes das 5 escolas profissionais, selecionados aleatoriamente de forma simples. Foi aplicado questionário de qualidade de sono de Pittsburgh. Resultados: A qualidade subjetiva de sono dos estudantes foi boa (47,1 \%) e má (42,5 \%), a latência do sono apresenta dificuldade ligeira (45,1 \%) e moderada (32 \%), a duração do sono foi superior a 5-6 horas (43,1\%), segue-se menos de 5 horas $(26,1 \%)$, a eficiência habitual do sono é inferior a $65 \%$ com uma percentagem de 90,8 \%, as perturbações do sono são classificadas como normais (50,3 \%) e diurnas marginais (46,4 \%), a disfunção diurna é moderada $(45,1 \%)$ e ligeiramente problemática (35,9 \%). Conclusão: As carreiras profissionais que têm nível de perturbação do sono e merecem cuidados e tratamento médico por ordem decrescente são Enfermagem (19 \%), Odontologia (17,6 \%), Farmácia e Bioquímica (16,3 \%), Obstetrícia (15\%) e Medicina (13,7\%).

Palavras-chave: estudantes de ciências da saúde, qualidade de sono, sono

\section{Introducción}

El sueño es considerado como un proceso complejo que involucra múltiples sistemas. Está considerado como el mejor de los descansos que un organismo puede tener, no solo por la recuperación energética que se realiza durante el mismo, sino por la cantidad de beneficios físicos y mentales que se desprenden de esta actividad. Es una función fisiológica indispensable para la vida, fundamental para garantizar el bienestar físico y emocional del individuo, así como la armonía con su medio externo.

El sueño es una función fisiológica importante para el adecuado funcionamiento físico, psicológico, cognoscitivo e intelectual. Al sueño se le reconoce como estado activo porque es una actividad eléctrica cerebral, es un estado activo porque la actividad eléctrica cerebral es un estado de conciencia dinámico en el que ocurren grandes modificaciones del funcionamiento del organismo como: cambios en la presión arterial, frecuencia cardiaca alta, y la respiración rápida, temperatura corporal, secreción hormonal entre otros. Una persona pasa durmiendo alrededor de un tercio de tiempo de su vida, el sueño es esencial para una noche de descanso y una adecuada salud.

Los trastornos del sueño constituyen uno de los problemas de salud más relevantes en las sociedades occidentales. La importancia de una buena calidad de sueño no solamente es fundamental como factor determinante de la salud, sino como elemento propiciador de una buena calidad de vida. La calidad del sueño no se refiere únicamente al hecho de dormir bien durante la noche, sino que también incluye un buen funcionamiento diurno (un adecuado nivel de atención para realizar diferentes tareas). La privación crónica de sueño genera alteraciones en el comportamiento, la atención, la memoria y el ánimo. El número de horas necesarias que un individuo promedio debe dormir diariamente para mantener un funcionamiento adecuado durante la vigilia se ha definido en 7 horas, lo cual es variable, porque existen dormidores cortos y largos; es decir, que requieren periodos de 6 horas para reponerse correctamente y otros que requieren más de 8 horas. Una buena calidad de sueño no solo está definida como el dormir bien durante la noche sino también a un desempeño adecuado durante la vigilia.

De este modo, la calidad del sueño constituye un aspecto clínico de enorme relevancia. Así lo demuestran las estadísticas al respecto: 30-40 \% de la población padece de insomnio, 1-10\% sufre apneas de sueño y $60 \%$ de los trabajadores por turnos informa de alteraciones del ritmo circadiano. ${ }^{1}$

Los estudiantes universitarios y en especial los que cursan estudios de ciencias de la salud tienen una elevada prevalencia de problemas del sueño debido a horarios irregulares, carga académica más intensa y turnos nocturnos. La frecuencia de trastornos del sueño es variable, encontrándola entre 50 y 70 $\%$. Estudios realizados en Perú señalan variaciones entre $45,7 \%$ y $58 \%$, esta elevada proporción de estudiantes de medicina con alteraciones del sueño requiere una particular atención, ya que la privación de sueño ha sido relacionada con detrimento de múltiples variables cognitivas, tales como la atención, memoria, velocidad de procesamiento y raciocinio.

Es conocido que, para muchos jóvenes, la universidad representa una forma de autonomía personal que no habían experimentado, en este contexto, se producen cambios en el estilo de vida, uno 
de los que se alteran con más frecuencia es el sueño. Tal vez debido a las demandas sociales y académicas, muchos estudiantes universitarios adoptan patrones de sueño irregulares, los cuales dan lugar a variación en los hábitos de sueño. A pesar de esto, la relación entre el sueño y las características de salud, bienestar y la somnolencia, han recibido poca atención en los estudiantes universitarios, una manera de estudiar dicha relación es clasificando el sueño en dos componentes, cantidad y calidad. Los componentes cuantificables son número de despertares nocturnos, latencia y duración del sueño; mientras que la calidad contiene índices en gran medida subjetivos del mismo, tales como, la profundidad del sueño, el descanso al despertar y la satisfacción general. ${ }^{2}$

Se conoce que los estudiantes universitarios, por su demanda académica, son una población de gran riesgo para desarrollar alteraciones en los patrones del sueño, a su vez, estos cambios acarrean consecuencias de estrés psicológico que repercuten en la salud mental del individuo, por lo tanto, las interrupciones en el sueño, asociadas a las demandas académicas podrían ser un predictor para las enfermedades de salud mental en esta población estudiantil. $^{3}$

Los estudiantes de la UNJBG y en especial los de ciencias de la salud tienen una elevada prevalencia de problemas del sueño debido a horarios irregulares, carga académica más intensa y turnos nocturnos.

La presente investigación fue desarrollada en las y los estudiantes de las distintas carreras de la Facultad de Ciencias de la Salud de la Universidad Nacional Jorge Basadre Grohmann de Tacna, que acoge a 5 carreras de la salud como son Medicina, Enfermería, Obstetricia, Odontología y Farmacia y Bioquímica.

El propósito de este estudio fue identificar la calidad de sueños de los estudiantes y determinar algunas características sociodemográficas de esta población en estudio.

\section{Material y métodos}

El presente estudio desarrolló el tipo de investigación descriptiva de cohorte transversal que permitió arribar a conclusiones específicas que serán de utilidad para una intervención oportuna, la muestra representativa fue 153 estudiantes de cada una de las cinco carreras profesionales de la Facultad de Ciencias de la Salud de la UNJBG. ${ }^{4}$
La técnica usada fue la encuesta y el instrumento fue el cuestionario Test de Calidad de Sueño de Pittsburgh aplicado a los estudiantes de la Facultad de Ciencias de la Universidad Nacional Jorge Basadre Grohmann. El índice de calidad de sueño de Pittsburgh es un índice ampliamente utilizado en clínica e investigación, este instrumento cuenta con 19 ítems que pretende valorar la mayor o menor calidad de sueño, durante el mes previo del individuo encuestado. Los ítems analizan los diferentes factores determinantes de la calidad de sueño a saber: calidad del sueño, latencia del sueño, duración del sueño, eficiencia del sueño, alteraciones del sueño, uso de medicación para dormir y disfunción diurna, la información obtenida se procesó mediante el paquete estadístico SPSS y se presenta en tablas de frecuencia de simple y doble entrada.

\section{Resultados}

En la Tabla 1 se aprecia la calidad subjetiva de sueño de estudiantes de la Facultad de Ciencias de la Salud, siendo en un $47,1 \%$ buena, mala en un $42,5 \%$, bastante mala en un $7,2 \%$ y bastante buena en un $3,3 \%$.

\section{Tabla 1}

Distribución, según calidad subjetiva de sueño

\begin{tabular}{lcc}
\hline $\begin{array}{l}\text { Calidad subjetiva } \\
\text { del sueño }\end{array}$ & $\mathrm{N} .^{\circ}$ & $\%$ \\
\hline Bastante buena & 5 & 3,3 \\
Buena & 72 & 47,1 \\
Mala & 65 & 42,5 \\
Bastante mala & 11 & 7,2 \\
\hline Total & 153 & 100 \\
\hline
\end{tabular}

En la Tabla 2, en cuanto a la latencia del sueño, se puede observar que el $45,1 \%$ de la población estudiantil presenta dificultad leve, y un 32 $\%$ dificultad moderada, facilidad $13,1 \%$ y dificultad severa $9,8 \%$. 
Tabla 2

Distribución, según latencia de sueño

\begin{tabular}{lcc}
\hline Latencia del sueño & $\mathrm{N}^{\circ}$ & $\%$ \\
\hline Facilidad & 20 & 13,1 \\
Dificultad leve & 69 & 45,1 \\
Dificultad moderada & 49 & 32 \\
Dificultad severa & 15 & 9,8 \\
\hline Total & 153 & 100 \\
\hline
\end{tabular}

En la Tabla 3 se aprecia que un $43,1 \%$ de los estudiantes tiene una duración del dormir de 5 a 6 horas; un $26,1 \%$, menos de 5 horas; un $23,5 \%$, de 6 a 7 horas; y menos de 7 horas, 7,2 \%.

Tabla 3

Distribución, según duración del dormir

\begin{tabular}{lcc}
\hline Duración del dormir & $\mathrm{N} .^{\circ}$ & $\%$ \\
\hline$>7$ horas & 11 & 7,2 \\
6-7 horas & 36 & 23,5 \\
$5-6$ horas & 66 & 43,1 \\
$<5$ horas & 40 & 26,1 \\
\hline Total & 153 & 100 \\
\hline
\end{tabular}

En la Tabla 4 se observa que el 90,8\% de los estudiantes presenta una eficiencia habitual del sueño de menos del $65 \%$; un $5,2 \%$, entre $65-74 \%$; un $3,3 \%$, entre $75-84 \%$; y un $0,7 \%$, en menos de un $85 \%$.

\section{Tabla 4}

Distribución, según eficiencia del sueño habitual

\begin{tabular}{lcc}
\hline $\begin{array}{l}\text { Eficiencia del sueño } \\
\text { habitual }\end{array}$ & $\mathrm{N} .{ }^{\circ}$ & $\%$ \\
\hline$>85 \%$ & 1 & 0,7 \\
$75-84 \%$ & 5 & 3,3 \\
$65-74 \%$ & 8 & 5,2 \\
$<65 \%$ & 139 & 90,8 \\
\hline Total & 153 & 100 \\
\hline
\end{tabular}

En la Tabla 5 se puede apreciar que el $50,3 \%$ de los estudiantes presenta alteraciones del sueño normal, seguida de un $46,7 \%$ de diurna marginal, un 2,6\% de alteraciones del sueño leve y un $0,7 \%$ diurna excesiva.

\section{Tabla 5}

Distribución, según alteraciones del sueño

\begin{tabular}{lcc}
\hline Alteraciones del sueño & $\mathrm{N} .{ }^{\circ}$ & $\%$ \\
\hline Leve & 4 & 2,6 \\
Normal & 77 & 50,3 \\
Diurna marginal & 71 & 46,4 \\
Diurna excesiva & 1 & 0,7 \\
\hline Total & 153 & 100 \\
\hline
\end{tabular}

En la Tabla 6 se observa que el $45,1 \%$ de los estudiantes presenta una disfunción diurna moderadamente, un 35,9 \% una disfunción diurna ligeramente problemático, un $12,4 \%$ muy problemático y un $6,5 \%$ nada problemático.

\section{Tabla 6}

Distribución, según disfunción diurna

\begin{tabular}{lcc}
\hline Disfunción diurna & $\mathrm{N} .{ }^{\circ}$ & $\%$ \\
\hline Nada problemático & 10 & 6,5 \\
Ligeramente & 55 & 35,9 \\
problemático & 69 & 45,1 \\
Moderadamente & 19 & 12,4 \\
Muy problemático & 153 & 100 \\
\hline Total &
\end{tabular}


En la Tabla 7, en cuanto al nivel de perturbación de sueño, el $81,7 \%$ merece atención y tratamiento médico, el 9,2\% presenta problema de sueño, el 8,5 $\%$ atención médica y el $0,7 \%$ sin problema de sueño.

\section{Tabla 7}

Distribución, según nivel de perturbación del sueño

\begin{tabular}{lcc}
\hline $\begin{array}{l}\text { Nivel de perturbación de } \\
\text { sueño }\end{array}$ & N. ${ }^{\circ}$ & $\%$ \\
\hline Sin problema de sueño & 1 & 0,7 \\
Merece atención médica & 13 & 8,5 \\
$\begin{array}{l}\text { Merece atención y tratamiento } \\
\text { médico }\end{array}$ & 125 & 81,7 \\
Problema de sueño & 14 & 9,2 \\
\hline Total & 153 & 100 \\
\hline
\end{tabular}

En la Tabla 8 se aprecia que las carreras profesionales que tienen nivel de perturbación de sueño y merecen atención y tratamiento médico son: Enfermería con un $19 \%$, Odontología con un 17,6 $\%$, Farmacia y Bioquímica con un $16,3 \%$, Obstetricia con un $15 \%$ y Medicina con un 13,7 \%.

\section{Discusión}

En el presente trabajo de investigación se realizó un estudio aplicando el cuestionario de Pittsburg de calidad de sueño, relacionando algunas características sociodemográficas en 153 estudiantes de las distintas carreras profesionales de la Facultad de Ciencia de la Salud de la UNJBG.

Las escuelas profesionales de los estudiantes participantes son Odontología, Obstetricia, Enfermería, Medicina y Farmacia y Bioquímica, con un promedio de 26 a 30 estudiantes por carrera seleccionados en forma aleatoria simple, y que en su mayoría estuvieron cursando el VI y IV semestre con un $35,9 \%$ y $24,2 \%$.

En cuanto a la calidad subjetiva del sueño se aprecia que casi el $50 \%$ de la población está divida en calidad buena $47,1 \%$ y calidad mala con un 42,5 $\%$ y un grupo de bastante mala con un $7,2 \%$ lo que se torna preocupante, y a la indagación refieren que son semestres donde se llevan cursos de carrera que necesitan mayor esfuerzo y dedicación. Al compararlo con otros estudios se aprecia que existe una cercana coincidencia con Paico Liñan al encontrar un $56,44 \%$ de internos de medicina con una calidad

Tabla 8

Distribución, según nivel de perturbación de sueño relacionado a la escuela profesional

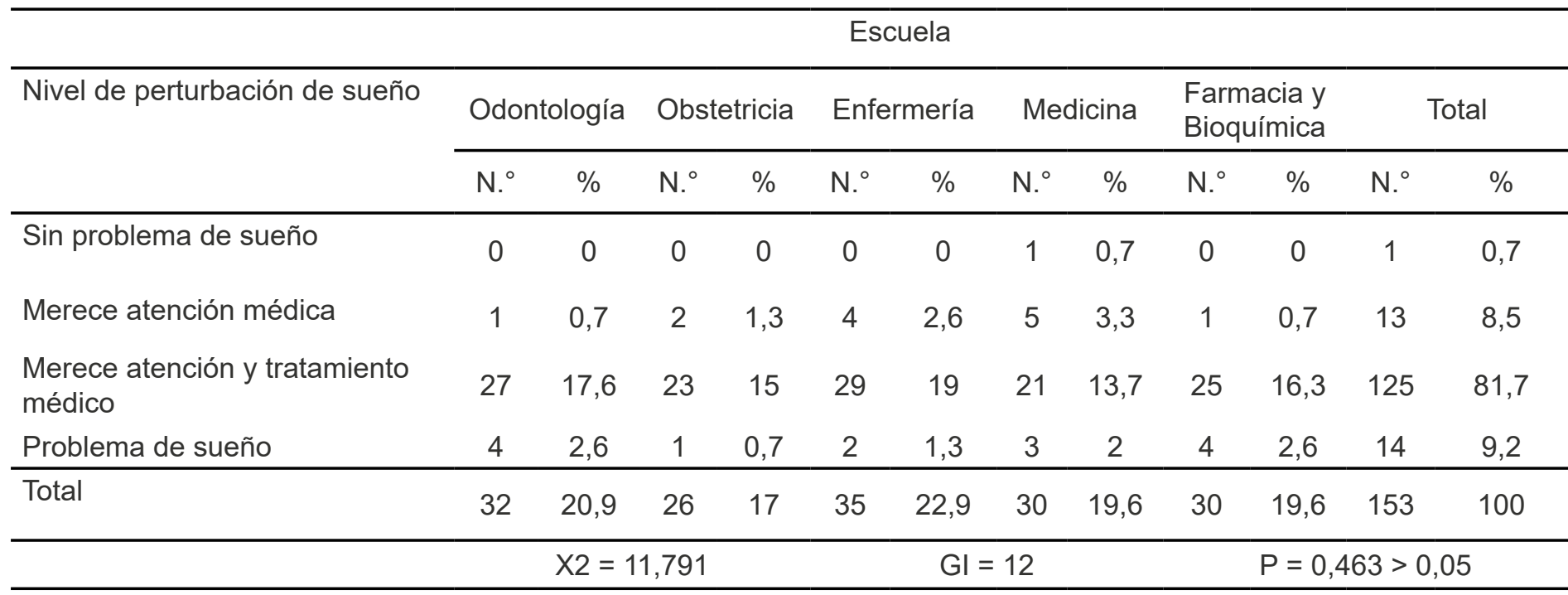


de sueño bastante mala, un 37,62 \% presentan una somnolencia Marginal o Moderada. La somnolencia y la calidad del sueño son variables subjetivas y están sujetas a variabilidad individual consideramos que tanto la escala de somnolencia Epworth como el cuestionario de Pittsburg son herramientas sencillas, económicas y útiles para calificar somnolencia y calidad de sueño en la población peruana. ${ }^{5}$

En cuanto a la latencia de sueño que según Pittsburg considera evaluar el tiempo de haber tardado en dormirse y cuántas veces no haber podido conciliar el sueño en la primera media hora, aquí se encontró que un $45,1 \%$ presenta dificultad leve y moderada con un $32 \%$, datos no coincidentes con los de Villarroel Prieto, en el 2013, en España, estudio realizado con estudiantes de medicina y enfermería donde reportan tener una fase de latencia buena y muy buena. ${ }^{6}$

En cuanto al componente duración del dormir, es uno de los que más afecta a estudiantes de carreras de la salud por la naturaleza de estas, la gran responsabilidad y el nivel de exigencia de ellas. En este estudio el $43,1 \%$ duerme entre 5 a 6 horas y un $26,1 \%$ menos de 5 horas, si bien es cierto, el número adecuado de horas debe ser de 8 , aquí no se cumple por las razones antes mencionadas, este resultado coincide también con lo reportado por Paico Liñan donde menciona que el componente duración es el que más afecta la calidad de sueño negativamente, siendo la tendencia a dormir unas 5-6 horas correspondiendo a un 40,59 \%. ${ }^{5}$

La eficiencia del sueño habitual, es el porcentaje de horas de sueño efectivo entre el número de horas que una persona permanece en la cama, en este estudio el 90,8\% de los estudiantes presentan menos del $65 \%$ lo que significa que casi la totalidad de los jóvenes no tiene una buena eficiencia habitual del sueño, este resultado difiere con los reportados por Quispe Mendoza en un estudio sobre somnolencia y calidad de sueño, ya que reporta en este componente una alta eficiencia en el sueño (mayor de $85 \%$ ) en estudiantes de enfermería de $\mathrm{V}$ y VI semestre. ${ }^{7}$

Pittsburgs también presenta un indicador de alteraciones del sueño, condición que impide que las personas duerman de manera normal tales como despertares nocturnos, alteraciones miccionales, frío, calor, pesadillas, etc. En esta investigación, el mayor porcentaje de la población de estudiantes está dentro de lo normal con un $50,3 \%$ y un $46,4 \%$ diurna marginal. Paico Liñan coincide de alguna manera puesto que el $69,3 \%$ presenta una perturbación de sueño bastante buena. ${ }^{5}$

La disfunción diurna donde se considera un nivel inadecuado para realizar diferentes tareas durante el día, encontramos que un 45,1\% presenta una disfunción diurna moderada y un 35,9 $\%$, ligeramente problemático; sin embargo, para Rosales, Egoavil, La Cruz, y Rey, la disfunción diurna en los estudiantes que están al inicio de la carrera no presentaron problema en un $67,8 \%(p=0,01)$. Lo cual en proporción es mayor a la que presentaron los cursantes a mediados, $11 \%$ presentaron algo de problema y, finalmente, $2,4 \%$, gran problema para mantenerse despiertos en el día. ${ }^{8}$

En relación al nivel de perturbación de sueño en forma general, el 81,7 \% merece atención y tratamiento médico, dato que llama la atención y lo comparamos con lo encontrado por De la Portilla Maya S, Dussán Lubert C, Montoya Londoño DM, Taborda Chaurra J, Nieto Osorio LS, en la primera variable la media de los estudiantes, sin discriminar por sexo, fue de 7,1; hallazgo que indica una calidad del sueño que merece atención médica. Asimismo, en cuanto a la somnolencia diurna, se evidenció una puntuación promedio de 10 puntos que permite señalar un rango de "severidad moderada". Adicionalmente se muestra una alta dispersión en los puntajes de calidad de sueño y somnolencia (coeficientes de variación alrededor del $40 \%$ ), indicando valores heterogéneos en estas variables. ${ }^{9}$

En cuanto a las carreras profesionales apreciamos que tienen nivel de perturbación de sueño y merecen atención y tratamiento médico son: Enfermería con un $19 \%$, Odontología con un $17,6 \%$, Farmacia y Bioquímica con un 16,3\%, Obstetricia con un $15 \%$ y Medicina con un 13,7 $\%$. La calidad del sueño no se refiere únicamente al hecho de dormir bien durante la noche, sino que incluye también un buen funcionamiento diurno (un adecuado nivel de atención para realizar diferentes tareas). Ello hace que sea fundamental el estudio de la incidencia de estos trastornos en distintos tipos de poblaciones. Sierra, Juan Carlos; Jiménez Navarro, Carmen; Martín Ortiz, Juan Domingo, evalúan la 
calidad subjetiva del sueño en una muestra de estudiantes universitarios sanos sin trastornos médicos ni psicológicos graves, y con un nivel de funcionamiento normal. El primer hecho relevante es que aproximadamente un tercio de la muestra presenta importantes dificultades del sueño; así, 31 $\%$ señala que su calidad de sueño es mala y $28 \%$ informa de una eficiencia de sueño inferior a $85 \%$, porcentaje que se considera límite para establecer un diagnóstico de insomnio. ${ }^{10}$

\section{Referencias}

1. American Psychiatric Association. Diagnostic and Statistical Manual of Mental Disorders. Cuarta Edición. Washington EEUU. 1994.

2. Pilcher, Ginter, Sadowsky. Sleep quality versus sleep quantity: relationships between sleep and measures of health, well-being and sleepiness in college students. 1997.

3. Sahraian y Javadpour. La interrupción del sueño y su correlación con la angustia psicológica entre estudiantes de medicina. Universidad de Ciencias Médicas. Shiraz Irán. 2010.

4. Canales F. de Alvarado y otros. Metodología de la Investigación. Manual para el desarrollo del Personal de Salud". Editorial Limusa, México. 1994.
5. Paico Liñan E. Calidad de sueño y somnolencia diurna en internos de medicina del Hospital Dos de Mayo periodo setiembre-noviembre 2015. Lima 2015

6. Villarroel Prieto V. Calidad de sueño en estudiantes de las carreras de medicina y enfermería, Universidad de los Andes Mérida Venezuela. 2013.

7. Quispe Mendoza W. Somnolencia y calidad de sueño en estudiantes de $\mathrm{V}$ y VI semestre de la Facultad de Enfermería de la Universidad del Altiplano Puno 2017.

8. Rosales E, Egoavil M, La Cruz C, Rey de Castro J. Somnolencia y calidad del sueño en estudiantes de medicina de una universidad peruana. 2007.

9. De la Portilla Maya S, Dussán Lubert C, Montoya Londoño DM, Taborda Chaurra J, Nieto Osorio LS. Calidad de sueño y somnolencia diurna excesiva en estudiantes universitarios de diferentes dominios.

10. Sierra JC, Jiménez N, Martín JD. Calidad del sueño en estudiantes universitarios: importancia de la higiene del sueño Salud Mental, 2002. México.

\section{Correspondencia:}

msilvac@unjbg.edu.pe
Fecha de recepción: 10/06/2021

Fecha de aceptación: 03/08/2021 